\title{
Infrared Spectroscopy of Imidazole Trimer in Helium Nanodroplets: Free NH Stretch Mode
}

\author{
Seulki Lee, Seung Jun Lee, Ahreum Ahn, Yusic Kim, Ahreum Min, Myong Yong Choi, ${ }^{*}$ and Roger E. Miller ${ }^{\dagger, a}$ \\ Department of Chemistry and Research Institute of Natural Science, Gyeongsang National University, Jinju 660-701, Korea \\ *E-mail:mychoi@gnu.ac.kr \\ 'Department of Chemistry, The University of North Carolina at Chapel Hill, Chapel Hill, North Carolina 27599, U.S.A. \\ Received January 4, 2010, Accepted January 6, 2011
}

\begin{abstract}
We report the first vibrational band assignment of imidazole trimer (IMT) solvated in helium nanodroplets. Several congested vibrational bands of imidazole (IM) clusters were obtained in the frequency region of 3513$3515 \mathrm{~cm}^{-1}$ and vibrationally resolved due to the extremely low temperature $(0.37 \mathrm{~K})$ and very weak solutesolvent interaction environments of helium droplets. The unambiguous free NH band assignment was achieved with an aid of pick-up oven temperature dependence and vibrational transition moment angle (VTMA) experiments as well as density functional theory (DFT) calculations. The band at $3514.3 \mathrm{~cm}^{-1}$ is attributed to the free NH stretching mode of linear IMT clusters, easily formed by the dipole-dipole interactions of IM in ultracold helium nanodroplets.
\end{abstract}

Key Words : Superfluid helium nanodroplets, Imidazole trimer, Density functional theory (DFT) calculations, Vibrational transition moment angles (VTMAs)

\section{Introduction}

Imidazole (IM), a five-membered heterocyclic ring structure acting as a proton donor and a proton acceptor, plays an important role in molecular conformations, protein folding and the hydrogen bonding interactions in biological systems. ${ }^{1}$ Therefore, IM and imidazole dimer (IMD) have been the subject of several investigations to get information about their intermolecular interactions. ${ }^{1-4}$ Despite its importance, to the best of our knowledge no spectroscopic information concerning the imidazole trimer (IMT) is known. Recently, we have reported infrared spectroscopic studies of IM, IMD and its water complexes (IMW and IMDW) in helium nanodroplets. ${ }^{3,4}$ In a continuing series of reports, we extend the experimental and theoretical studies of IM clusters to IMT.

In recent years there have been considerable advances in using helium nanodroplets. The very weak interactions between the target molecules and the helium give rise to almost unperturbed environments from that of the gas phase. Furthermore, the temperature of the helium nanodroplets is extremely low, $0.37 \mathrm{~K}$, thus the spectral broadening is significantly reduced making the He droplets as an ideal matrix for infrared spectroscopy.,

Using the high resolution spectra obtained from the above mentioned experimental conditions, we were able to measure the angle between the transition and permanent dipole moments of the target molecules, called the vibrational transition moment angle (VTMA), which turned out to be a very sensitive structural and unambiguous specific vibrational band assignment tool. Furthermore, VTMAs can be

${ }^{\mathrm{a}}$ Deceased. November 6, 2005. University of North Carolina at Chapel Hill also easily calculated and compared to the experimental value obtained in this study. The use of VTMAs for the specific tautomer and vibrational band assignments of small biomolecules is well described in our previous reports. ${ }^{3,4,6-12}$ In this paper we present the first vibrational band assignment of IMT in the free NH stretching region applying the VTMA techniques in He nanodroplets.

\section{Experimental and Density Functional Theory Calculations}

Infrared spectra of IM clusters, isolated in He nanodroplets, have been obtained using the apparatus described in detail elsewhere, ${ }^{6,13}$ and only a brief description of the apparatus is given here. He nanodroplets with a mean size of $3000 \mathrm{He}$ atoms were formed by supersonic expansion of ultrahigh pure helium (99.9999\%) from a $5 \mu \mathrm{m}$ diameter orifice, operated at 50 atm pressure and a temperature of 20 $\mathrm{K}$. The log-normal distribution of droplet sizes determined from published scaling laws ${ }^{13}$ for various source conditions was used in this study.

Similar to the previous IM and IMD studies, ${ }^{3,4}$ we have used the thermal evaporation method, using an effusive oven of IM (Aldrich, 99\% purity), to be picked up by He droplets. The number of IM molecules picked up by He droplets was adjusted by varying the oven temperature such that the associated Poisson distribution was employed to determine the cluster sizes. ${ }^{14}$ The optimized temperature of IM with the effusive oven for IMT was $\sim 55^{\circ} \mathrm{C}$. Upon being captured by the droplets, the IM molecules are rapidly cooled to the temperature of the droplets $(0.37 \mathrm{~K})$ and forming IM clusters inside the droplets.

Once the clusters of IM are formed in He droplets, the doped droplets are crossed many times with the IR beam 
generated from a periodically polled lithium niobate (PPLN) cw-OPO (Linos Photonics) in the multipass cell region. Therefore, the vibrational relaxation causes evaporation of the corresponding number of $\mathrm{He}$ atoms. Since we also know that the dipole moment of a solvated polar molecule can be strongly oriented by a large direct current (DC) electric field, we positioned electrodes on either side of the multipass cell. Then, the laser electric field direction was aligned either parallel or perpendicular to the DC electric field. When the applied field orients the dipole moment of a molecule, the excitation efficiency depends upon the direction and magnitude of both the vibrational transition moment and the laser electric field. As a result the angle between the vibrational transition moment and the permanent electric dipole moment, VTMA, could be obtained by measuring the band intensity ratio, $\mathrm{I}_{\text {para }} / \mathrm{I}_{\text {perp. }}$. A quantitative analysis of the angle requires a normalized permanent dipole moment orientation distribution, which is developed by Kong and coworkers ${ }^{15,16}$ and well described elsewhere. ${ }^{6-8,17}$

The experimental VTMAs can be compared directly with those obtained from density functional theory (DFT) calculations, based on full geometry optimizations and vibrational analysis using Gaussian 03. ${ }^{18}$ Unless otherwise specified, the calculations reported here were conducted at the B3LYP level using the $6-311++\mathrm{G}(\mathrm{d}, \mathrm{p})$ basis set.

\section{Results and Discussion}

Although a number of quantum mechanical calculations have been reported for IM, it is interesting to note that not many calculations on $\mathrm{IMD}^{19}$ and rarely any calculations on IMT have been reported. ${ }^{20}$ Furthermore, VTMAs of each system have not been included in any of them. Therefore, we carried out DFT calculations on the IMT complexes to obtain the VTMA and frequency of free NH stretching mode. As a result, the directions of calculated transition

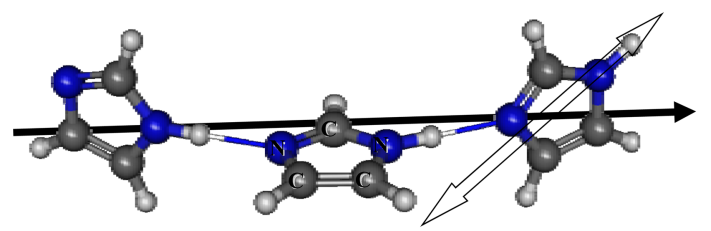

Figure 1. Calculated structure of imidazole timer (IMT) with the directions of the permanent electric dipole moment (solid arrow) and the vibrational transition moment of free $\mathrm{NH}$ mode (empty double ended arrow).

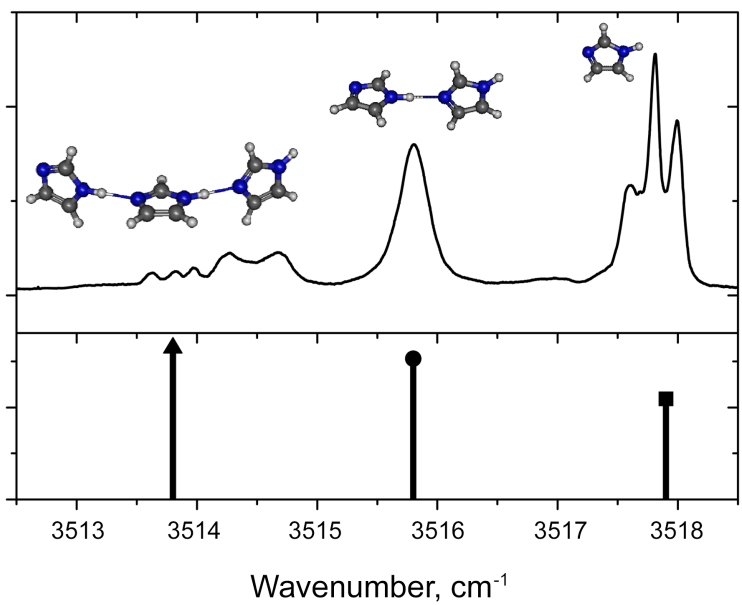

Figure 2. Infrared spectrum of imidazole monomer (IM), dimer (IMD) and trimer (IMT) in free NH vibrational stretching region in He droplets. The corresponding bar spectra for IM (square), IMD (circle) and IMT (triangle) scaled by a factor of $0.96265,0.96215$ and 0.9624, respectively, with B3LYP/6-311++G(d,p) basis set are shown below the experimental spectrum.

moment (empty double ended arrow) and permanent electric dipole moment (solid arrow) are plotted onto the structure of IMT in Figure 1.

The upper panel in Figure 2 shows an experimental spectrum of IM and its clusters in the region of free $\mathrm{NH}$ stretching vibrations and the corresponding structures. The bottom panel shows the calculated bar spectra for IM, IMD and IMT of which frequencies were scaled individually to take into account for their anharmonicity. Previously, the partially resolved PQR band at $3517.9 \mathrm{~cm}^{-1}$ and a broad band at $3515.8 \mathrm{~cm}^{-1}$ were assigned to the free $\mathrm{NH}$ band of IM and IMD, respectively., ${ }^{3,4}$ The obtained harmonic frequencies for the two bands were 3654.4 and $3654.1 \mathrm{~cm}^{-1}$, respectively, as shown in Table 1 . Therefore, the calculated frequencies were scaled for IM and IMD by a factor of 0.96265 and 0.96215 , respectively. Since the exact experimental frequency of the free NH stretching band for IMT is unknown at the moment, we have naively used the averaged scaling factor of IM and IMD, i.e., 0.9624, resulting in $3513.8 \mathrm{~cm}^{-1}$. In the previous studies, the free NH stretching band assignments for IM and IMD are quite straightforward, given that the predicted frequencies give a convincing assignment for the corresponding single band, however, for the case of IMT, there are several bands in the predicted

Table 1. A summary of the experimental and calculated data for IM, IMD and IMT

\begin{tabular}{ccccccc}
\hline Imidazole & $\begin{array}{c}\text { Exp. Freq. } \\
\left(\mathrm{cm}^{-1}\right)\end{array}$ & $\begin{array}{c}\text { Harm. Freq. }{ }^{a} \\
\left(\mathrm{~cm}^{-1}\right)\end{array}$ & Scaling Factor ${ }^{b}$ & $\begin{array}{c}\text { Scaled Freq. } \\
\left(\mathrm{cm}^{-1}\right)\end{array}$ & $\begin{array}{c}\text { DFT Intensity } \\
(\mathrm{km} / \mathrm{mol})\end{array}$ & $\begin{array}{c}\text { Dipole Moment } \\
(\text { Debye })\end{array}$ \\
\hline IM & 3517.9 & 3654.4 & 0.96265 & 3517.9 & 54.7 & 3.8 \\
IMD & 3515.8 & 3654.1 & 0.96215 & 3515.8 & 76.5 & 9.3 \\
IMT & 3514.3 & 3651.1 & 0.96240 & 3513.8 & 99.1 & 14.8 \\
\hline
\end{tabular}

$\overline{a^{a}}$ The calculations were performed at the B3LYP/6-311++G(d,p) level. ${ }^{b}$ The scaled frequencies for IM, IMD and IMT were obtained by multiplying the harmonic frequencies by a factor of $0.96265,0.96215$ and 0.9624 , respectively, (see the text) to account for the effects of anharmonicity. 


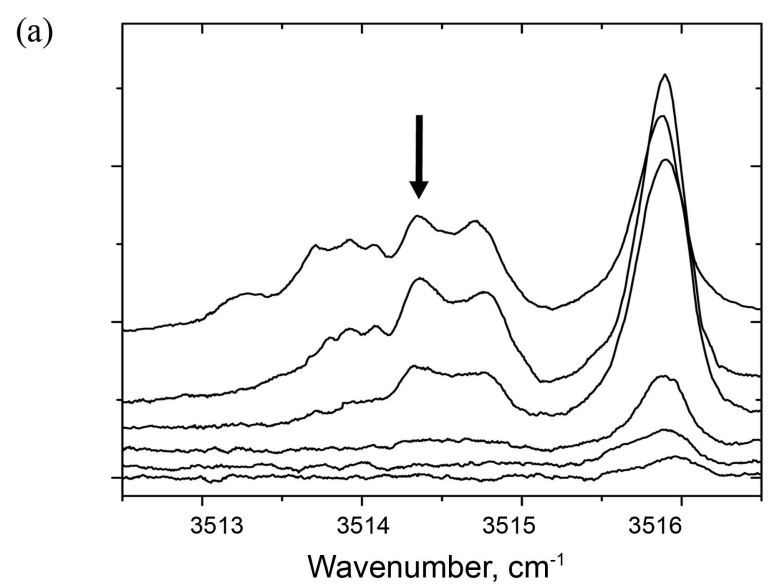

(b)

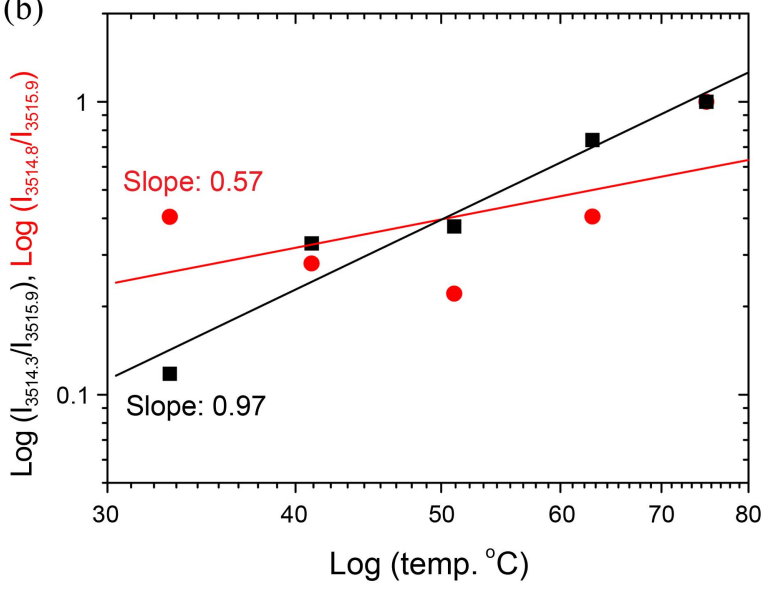

Figure 3. (a) Evolution of the free NH spectral region for different pick-up oven temperatures (top to bottom: 75, 63, 51, 41, 33 and 25 $\left.{ }^{\circ} \mathrm{C}\right)$. The band marked with a downwards arrow is the free $\mathrm{NH}$ stretch of imidazole trimer. (b) Log-log plots of the $\mathrm{I}_{3514.3} / \mathrm{I}_{3515.9}$ (square) and $\mathrm{I}_{3514.8} / \mathrm{I}_{3515.9}$ (circle) versus the pick-up oven temperatures.

frequency region at around $3513.8 \mathrm{~cm}^{-1}$ shown in Figure 2, which makes the band assignment of IMT difficult with frequency calculations alone. The calculated and scaled frequencies for IM, IMD and IMT using a B3LYP/6$311++\mathrm{G}(\mathrm{d}, \mathrm{p})$ basis set are summarized in Table 1 , along with permanent dipole moments and scaled harmonic frequencies.

To identify an IMT band among the congested bands, we have recorded the spectra at different pick-up oven temperatures from $25-75{ }^{\circ} \mathrm{C}$, shown in Figure 3(a). It is apparent that higher oven temperatures give rise to higher IM vapor pressures in the pick-up oven and thus a more probability of picking up more than one molecule. In general, the band assignments to the specific cluster size of IM are based on the pick-up oven temperature dependence of the corresponding peak intensities. ${ }^{4,-9,11}$ However, in this work, monitoring the peak intensity of the bands versus the pickup oven temperatures, shown in Figure 3(a), becomes unreliable, because a broad feature appears in the IMT band region as the oven temperature increases. Therefore, we have plotted the intensity ratio, $\mathrm{I}_{3514.3} / \mathrm{I}_{3515.9}$ and $\mathrm{I}_{3514.8} / \mathrm{I}_{3515.9}$, versus the oven temperatures instead, shown in the log-log plots of Figure 3(b), of which method is well described in

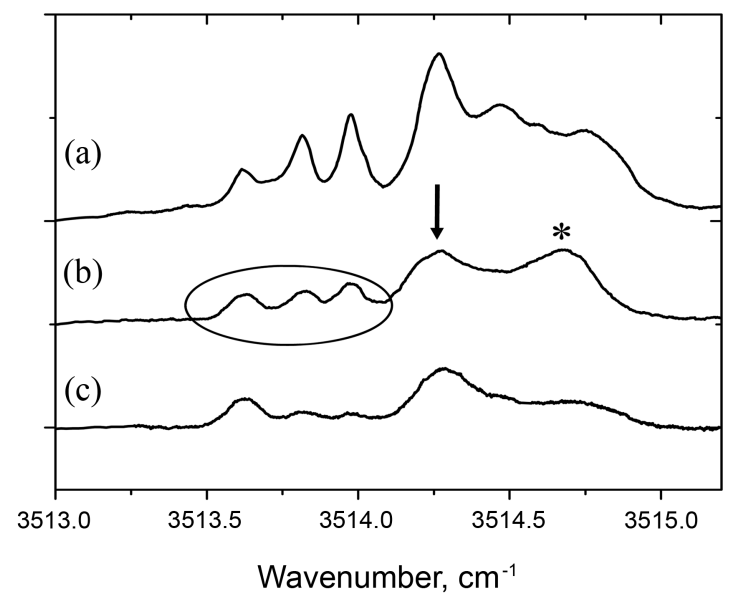

Figure 4. An expanded view of the free NH vibrational stretching region of higher order imidazole clusters. Infrared spectra (a), (b) and (c) correspond to parallel polarization, zero field and perpendicular polarization, respectively. The higher frequency band marked with an asterisk is composed of several bands identified in parallel polarization scan (a). The band at $3514.3 \mathrm{~cm}^{-1}$ marked with the downwards arrow is attributed to the free $\mathrm{NH}$ stretch mode of imidazole trimer. The bands inside the oval are possibly associated with the higher order clusters.

Ref. 21. Briefly, $\mathrm{I}_{3515.9}$ is the intensity of IMD as a known reference band and $I_{3514.3}$ and $I_{3514.8}$ are the intensity of unknown bands. The slopes of $\mathrm{I}_{3514.3} / \mathrm{I}_{3515.9}$ and $\mathrm{I}_{3514.8} / \mathrm{I}_{3515.9}$ are 0.97 and 0.57 , which indicates that the former band at $3514.3 \mathrm{~cm}^{-1}$ originates from clusters having three IM molecules, since the reference band is two IM molecules (IMD). The latter band at $3514.8 \mathrm{~cm}^{-1}$, however, shows a larger deviation from the integer values, indicating a presence of some overlapping bands underneath the band.

Now we think that the band at $3514.3 \mathrm{~cm}^{-1}$, marked with the downwards arrow in Figure 3(a), is the free NH stretching band of IMT from the oven temperature dependence experiments. To further support the band assignment of IMT, we have conducted electric field dependence experiments in the congested band region, in which the spectra were measured with (a) parallel polarization, (b) zero field, and (c) perpendicular polarization shown in Figure 4. It is instantaneously noticed that there are several bands underneath the band marked with an asterisk in the parallel polarization scan, (see Figure 4(a)). It is now clear that the several bands underneath the band marked with an asterisk are the cause of the large deviation in the band intensity ratios mentioned above.

Similar to the formation of linear chains of polar molecules, such as $\mathrm{HCN}$, in He droplets, ${ }^{22}$ the sequential addition of IM makes the IM clusters linear assembling head-to-tail because the existing chain, IMD, further induces the dipole oriented structure, i.e., IMT, IMQ, and so on. This can be possible that the dipole induced linear structures of IM having a large dipole moment $(3.8 \mathrm{D})$ are trapped in their potential well in the ultra low temperature environment. Basically, the length of linear IM cluster chains is restricted to the size of He droplets, because the large droplets have 
large cross sections and sufficiently high heat capacities to capture and cool many IM molecules. ${ }^{3}$ The lower frequency bands marked with an oval could be from the higher order clusters due to the rather smooth frequency evolution of the bands, however, the assignments of these bands are beyond the scope of the present work.

VTMA analysis of the band at $3514.3 \mathrm{~cm}^{-1}$ marked with a downwards arrow yielded an experimental value of $42^{\circ}$ while the calculated VTMA for the band is $40^{\circ}$. This shows that the VTMA assignment between experiment and theory is clearly excellent. This is important given that an unambiguous assignment could not be obtained by only comparing the values between experiments and frequency calculations, given that the band region is highly congested, making the band assignment of IMT questionable. Furthermore, the congested band region was composed of a broad band when the matrix of argon or even neon was used, of which typical spectral resolution of the traditional matrix-isolation spectroscopy is often limited even at low temperatures. ${ }^{23}$ Once again, the ultralow temperature of He droplets, providing the necessary cooling for such IR spectroscopic studies of large IM clusters, and the weak interactions between the superfluid helium and the target molecules result in high resolution and small vibrational frequency shifts. Moreover, several bands underneath the band marked with an asterisk are only identified at parallel electric field polarization, which otherwise the band assignment would be more difficult and/or ambiguous.

\section{Conclusions}

In this study we report high resolution infrared laser spectra of imidazole trimer (IMT) isolated in helium nanodroplets in the free $\mathrm{NH}$ stretching vibrational band region for the first time. The unambiguous band assignment of IMT solvated in helium nanodroplets was conducted by using several unique techniques, such as pick-up oven temperature and electric field dependence experiments. By varying the oven temperatures and measuring the band intensity ratios of unknown bands to the reference band (here IMD), we have been able to assign the band at $3514.3 \mathrm{~cm}^{-1}$ to the free $\mathrm{NH}$ stretch mode of IMT. Furthermore, by applying a large DC electric field and measuring the band intensity ratios of the corresponding band in parallel and perpendicular laser electric field, we achieved a definitive assignment for the free NH band of IMT. The IMT clusters in helium droplets are self-assembled by long-range dipole-dipole forces and form noncovalently bonded linear chains. We hope that this study shed lights on the further investigation on the formation of branched or ring structures of IM clusters when a certain number of IM is added to the linear chain structures.

Acknowledgments. This work is supported by the Korea Research Foundation (KRF) grant funded by the Korean
Government (MEST) (No. 2009-0074609 and 20090066270) and (No. R01-2008-000-20002-0(2008)), in which main calculations were performed by using the supercomputing resource of the Korea Institute of Science and Technology Information (KISTI).

\section{References}

1. Poterya, V.; Profant, V.; Farnik, M.; Sistik, L.; Slavicek, P.; Buck, U. J. Phys. Chem. A 2009, 113, 14583.

2. Carles, S.; Lecomte, F.; Schermann, J. P.; Desfrancois, C. J. Phys. Chem. A 2000, 104, 10662.

3. Choi, M. Y.; Miller, R. E. J. Phys. Chem. A 2006, 110, 9344.

4. Choi, M. Y.; Miller, R. E. Chem. Phys. Lett. 2009, 477, 276.

5. Toennies, J. P.; Vilesov, A. F. Angew. Chem. Int. Ed. 2004, 43, 2622.

6. Choi, M. Y.; Douberly, G. E.; Falconer, T. M.; Lewis, W. K.; Lindsay, C. M.; Merritt, J. M.; Stiles, P. L.; Miller, R. E. Int. Rev. Phys. Chem. 2006, 25, 15.

7. Choi, M. Y.; Dong, F.; Miller, R. E. Phil. Trans. R. Soc. A 2005, 363, 393.

8. Choi, M. Y.; Miller, R. E. J. Am. Chem. Soc. 2006, 128, 7320.

9. Choi, M. Y.; Miller, R. E. J. Phys. Chem. A 2007, 111, 2475.

10. Choi, M. Y.; Dong, F.; Han, S. W.; Miller, R. E. J. Phys. Chem. A 2008, 112, 7185 .

11. Lee, S. J.; Choi, M. Y.; Miller, R. E. Chem. Phys. Lett. 2009, 475, 24.

12. Min, A.; Lee, S. J.; Choi, M. Y.; Miller, R. E. Bull. Korean Chem. Soc. 2009, 30, 3039.

13. Nauta, K.; Miller, R. E. J. Chem. Phys. 1999, 111, 3426.

14. Lewerenz, M.; Schilling, B.; Toennies, J. P. J. Chem. Phys. 1995, 102,8191 .

15. Franks, K. J.; Li, H. Z.; Kong, W. J. Chem. Phys. 1999, 110, 11779.

16. Kong, W.; Bulthuis, J. J. Phys. Chem. A 2000, 104, 1055.

17. Douberly, G. E.; Miller, R. E. J. Phys. Chem. B 2003, 107, 4500.

18. Frisch, M. J.; Trucks, G. W.; Schlegel, H. B.; Scuseria, G. E.; Robb, M. A.; Cheeseman, J. R.; Montgomery, J. J. A.; Vreven, T.; Kudin, K. N.; Burant, J. C.; Millam, J. M.; Iyengar, S. S.; Tomasi, J.; Barone, V.; Mennucci, B.; Cossi, M.; Scalmani, G.; Rega, N.; Petersson, G. A.; Nakatsuji, H.; Hada, M.; Ehara, M.; Toyota, K.; Fukuda, R.; Hasegawa, J.; Ishida, M.; Nakajima, T.; Honda, Y.; Kitao, O.; Nakai, H.; Klene, M.; Li, X.; Knox, J. E.; Hratchian, H. P.; Cross, J. B.; Adamo, C.; Jaramillo, J.; Gomperts, R.; Stratmann, R. E.; Yazyev, O.; Austin, A. J.; Cammi, R.; Pomelli, C.; Ochterski, J. W.; Ayala, P. Y.; Morokuma, K.; Voth, G. A.; Salvador, P.; Dannenberg, J. J.; Zakrzewski, V. G.; Dapprich, S.; Daniels, A. D.; Strain, M. C.; Farkas, O.; Malick, D. K.; Rabuck, A. D.; Raghavachari, K.; Foresman, J. B.; Ortiz, J. V.; Cui, Q.; Baboul, A. G.; Clifford, S.; Cioslowski, J.; Stefanov, B. B.; Liu, G.; Liashenko, A.; Piskorz, P.; Komaromi, I.; Martin, R. L.; Fox, D. J.; Keith, T.; Al-Laham, M. A.; Peng, C. Y.; Nanayakkara, A.; Challacombe, M.; Gill, P. M. W.; Johnson, B.; Chen, W.; Wong, M. W.; Gonzalez, C.; and Pople, J. A. Gaussian, Inc., Wallingford CT, 2004.

19. Yan, S.; Bu, Y. J. Phys. Chem. B 2004, 108, 13874.

20. Torrent, M.; Musaev, D. G.; Morokuma, K.; Ke, S. C.; Warncke, K. J. Phys. Chem. B 1999, 103, 8618.

21. Flynn, S. D.; Skvortsov, D.; Morrison, A. M.; Liang, T.; Choi, M. Y.; Douberly, G. E.; Vilesov, A. F. J. Phys. Chem. Lett. 2010, 1, 2233.

22. Nauta, K.; Miller, R. E. Science 1999, 283, 1895.

23. Van Bael, M. K.; Smets, J.; Schoone, K.; Houben, L.; McCarthy, W.; Adamowicz, L.; Nowak, M. J.; Maes, G. J. Phys. Chem. A 1997, 101, 2397. 